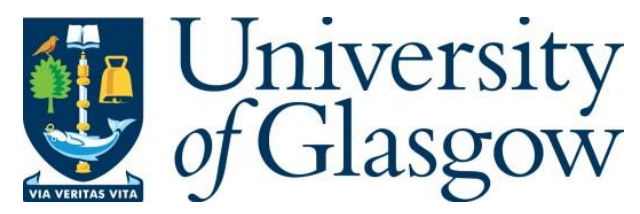

Di Campli San Vito, P., Brown, E., Brewster, S., Pollick, F., Thompson, S., Skrypchuk, L. and Mouzakitis, A. (2020) Haptic Feedback for the Transfer of Control in Autonomous Vehicles. In: Automotive UI '20 Adjunct, Virtual Event, USA, 21-22 Sep 2020, pp. 34-37. ISBN 9781450380669

(doi: 10.1145/3409251.3411717).

There may be differences between this version and the published version. You are advised to consult the publisher's version if you wish to cite from it.

(C) The Authors 2020. This is the author's version of the work. It is posted here for your personal use. Not for redistribution. The definitive Version of Record was published in Proceedings of Automotive UI '20 Adjunct, Virtual Event, USA, 2122 Sep 2020, pp. 34-37. ISBN 9781450380669.

http://eprints.gla.ac.uk/222430/

Deposited on: 18 August 2020

Enlighten - Research publications by members of the University of Glasgow http://eprints.gla.ac.uk 


\section{Haptic Feedback for the Transfer of Control in Autonomous Vehicles}

\author{
Patrizia Di Campli San Vito \\ p.di-campli-san- \\ vito.1@research.gla.ac.uk \\ School of Computing Science, \\ University of Glasgow \\ Glasgow, United Kingdom \\ Frank Pollick \\ frank.pollick@glasgow.ac.uk \\ School of Psychology, University of \\ Glasgow \\ Glasgow, United Kingdom
}

\author{
Edward Brown \\ ebrown6@jaguarlandrover.com \\ Jaguar Land Rover Ltd. \\ Coventry, United Kingdom
}

Simon Thompson

sthom261@jaguarlandrover.com

Jaguar Land Rover Ltd.

Coventry, United Kingdom

\author{
Stephen Brewster \\ stephen.brewster@glasgow.ac.uk \\ School of Computing Science, \\ University of Glasgow \\ Glasgow, United Kingdom
}

\author{
Lee Skrypchuk \\ lskrypchuk@jaguarlandrover.com \\ Jaguar Land Rover Ltd. \\ Coventry, United Kingdom
}

\section{Alexandros Mouzakitis \\ amouzak1@jaguarlandrover.com \\ Jaguar Land Rover Ltd. \\ Coventry, United Kingdom}

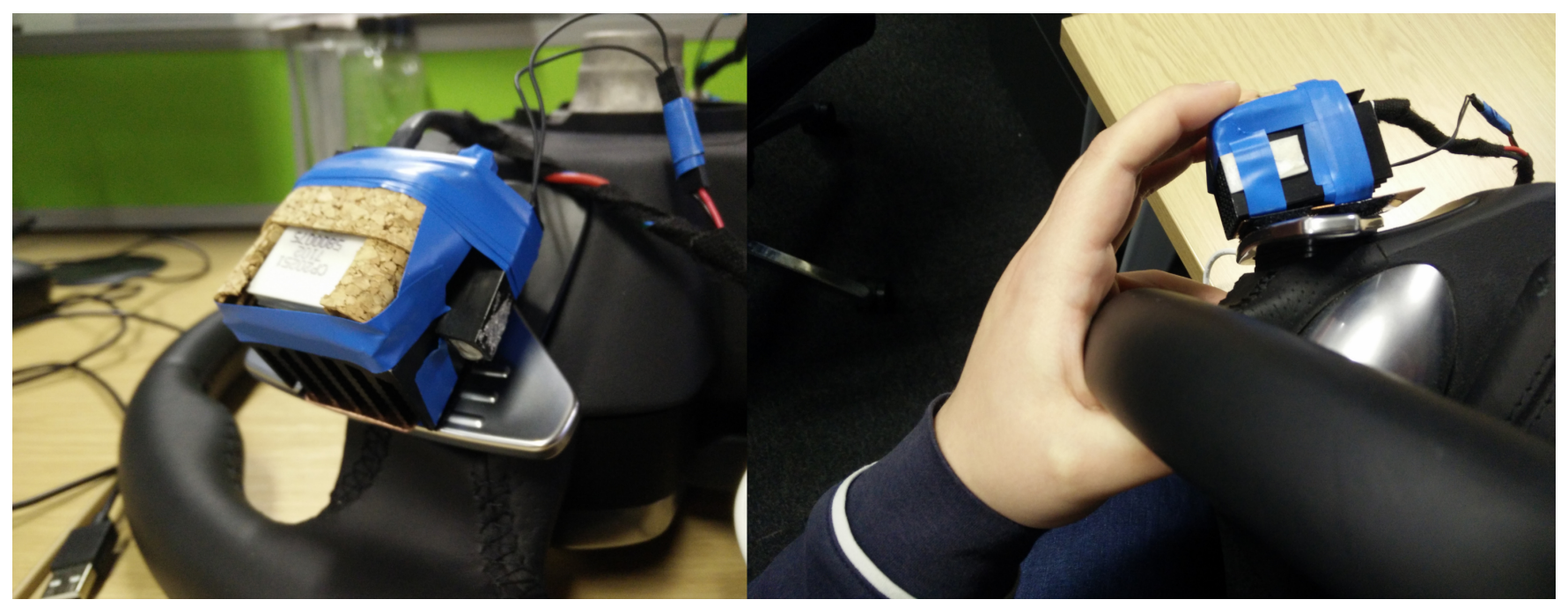

Figure 1: Haptic devices attached to the gear paddles.

\begin{abstract}
Vehicles offering autonomous features need effective methods for transferring the control from the driver to the vehicle and back. While most research focuses on presenting information the driver might need after retaking control, our study investigates ways to

Permission to make digital or hard copies of part or all of this work for personal or classroom use is granted without fee provided that copies are not made or distributed for profit or commercial advantage and that copies bear this notice and the full citation on the first page. Copyrights for third-party components of this work must be honored. For all other uses, contact the owner/author(s).

AutomotiveUI '20 Adjunct, September 21-22, 2020, Virtual Event, USA

(c) 2020 Copyright held by the owner/author(s).

ACM ISBN 978-1-4503-8066-9/20/09.

https://doi.org/10.1145/3409251.3411717
\end{abstract}

improve the process of transferring control itself. We investigated multimodal feedback with and without haptics and visuals in a simulator study. Results showed that visual and haptic feedback improved driving during handover. Subjective ratings described multimodal feedback without visual as more disruptive than with the visual feedback included. Furthermore, ratings showed a preference for including visual and haptic feedback. These results lead us a step closer to a safe, clear and accepted control transfer process between driver and vehicle.

\section{CCS CONCEPTS}

- Human-centered computing $\rightarrow$ Haptic devices. 


\section{KEYWORDS}

Thermal; vibrotactile; haptic; in-car; feedback; transfer of control; autonomous vehicle

\section{ACM Reference Format:}

Patrizia Di Campli San Vito, Edward Brown, Stephen Brewster, Frank Pollick, Simon Thompson, Lee Skrypchuk, and Alexandros Mouzakitis. 2020. Haptic Feedback for the Transfer of Control in Autonomous Vehicles. In 12th International Conference on Automotive User Interfaces and Interactive Vehicular Applications (AutomotiveUI '20 Adjunct), September 21-22, 2020, Virtual Event, USA. ACM, New York, NY, USA, 4 pages. https: //doi.org/10.1145/3409251.3411717

\section{INTRODUCTION}

Increasing numbers of cars include autonomous features, transferring lateral and/or velocity control to the vehicle. These features must be activated (handover of control to the car) and deactivated (takeover of control from the car), and are often only available for specific situations, such as motorway driving. It is vital that drivers are aware of the state of autonomy and are well informed during the process of transferring control. Research into control transfer mostly investigated takeover requests, especially in emergency situations, where the vehicle gives control back to the driver in dangerous situations and the driver needs to react quickly and accurately to avert accidents. Haptic feedback in these situations was introduced to inform drivers of obstacles to avoid after takeover [7,9] and/or improve takeover time $[6,8,10]$. While there is a wide body of research on control transfer, using haptics to indicate the status of the transfer itself has been rarely tested.

In pilot tests we observed participants not releasing the steering wheel or the accelerator pedal after activating autonomous features, indicating their uncertainty of when the control had been fully transferred to the car. Haptic feedback on these locations could guide and reassure drivers. While Salminen et al.[10] explored multimodal feedback for control transfer, they focused on reaction times after takeover and the influence of a secondary task. In the past, we presented unimodal thermal feedback during driving for directional information [2,3], but it has not yet been used for notifications or in a multimodal setting.

Commonly, single button presses $[4,12]$ or lever pulls $[9,13]$ are used to transfer control. However, these actions may be done unintentionally, potentially leading to dangerous situations with drivers incorrectly assuming the vehicle is driving autonomously. Simultaneous double button press [1] can overcome this, however, in pilot studies we observed that searching for correct buttons led to long glances away from the road and high off-the-road times, increasing the risk of crashes [5]. Therefore, we tested simultaneous gear paddle pull, as envisioned by Volvo ${ }^{1}$. Additionally, eyes-offthe-road time can also be reduced by using non-visual feedback for unfamiliar in-car interactions [11].

Therefore, we explored the benefits of thermal and vibrotactile feedback for control transfer with a double paddle pull in a user study.

\footnotetext{
${ }^{1}$ https://www.media.volvocars.com/global/en-gb/media/pressreleases/167739/volvo cars-reveals-safe-and-seamless-user-interface-for-self-driving-cars (accessed $22 / 10 / 2019$
}

\section{STUDY DESIGN}

The within-subjects experiment had two Independent Variables: haptic and visual. The combinations, or feedback types, investigated were:

- Visual progress bar, without haptics (NoHapVis)

- Visual progress bar, with haptics (HapVis)

- No visual progress bar, with haptics (HapNoVis)

The autonomous driving feature, taking over full control, was activated and deactivated by simultaneously pulling both gear paddles for 2 s. Building on feedback tested in pilot studies, sound and a visual icon on the instrument cluster behind the steering wheel informed the driver when the autonomous feature was available and speech indicated its activation. In the deactivation process, sound feedback signalled the start of the transfer and speech its completion. The haptic feedback consisted of thermal and vibrotactile cues. During manual driving, the temperature of thermal devices on the paddles was set to $32^{\circ} \mathrm{C}$. While pulling the paddles, this temperature changed to $26^{\circ} \mathrm{C}$ with $3{ }^{\circ} \mathrm{C} / \mathrm{s}$. The process finished after $2 \mathrm{~s}$. Haptic devices on both gear paddles as well as the accelerator pedal vibrated for $0.5 \mathrm{~s}$ to mark the completed transfer and to remind the driver to release wheel and pedals. Process and feedback for deactivation were similar, only the temperature changed back to $32^{\circ} \mathrm{C}$.

The simulator drive was the same for all transfer types: participants were asked to stay within the middle lane at $40 \mathrm{mph}$. After about 90 s traffic intensified, leading to a decrease in speed, and about $30 \mathrm{~s}$ later the autonomous feature became available. The participants would then activate the feature and were then presented with $2 \mathrm{~min}$ movie snippets on the centre console. Afterwards, the experimenter asked participants to retake control and drive manually for $2 \mathrm{~min}$. Order of the conditions and videos across the transfer types were counterbalanced.

Driving performance was measured by evaluating simulator log files from availability of the autonomous feature until activation. The simulator logged changes in steering behaviour, indicating distraction through higher number of logging entries or steering occurrences. Lane deviation was calculated as Root Mean Square Error of the car's position compared to the middle of the lane. At deactivation the time just after regaining control was examined.

Driving behaviour was compared between and within feedback conditions: the driving during the control transfer sequence was compared to a baseline taken just before the autonomous feature became available. As the takeover occurred $2 \mathrm{~min}$ after the baseline, road conditions had significantly changed and could not be compared.

Gaze behaviour was investigated by manually labelling videos recorded during driving. Overall eyes-off-the-road-time, time taken to release wheel and pedals and long glances over 2 s were marked by the experimenter. Participants filled in questionnaires at the end of the each driving block, measuring perceived workload (NASA TLX) and preference ratings.

We hypothesised that adding haptic feedback would help drivers to release wheel and pedals faster, as the localised vibration declared the exact moment control was transferred. As transfer progress information was translated to temperature change, we were interested to see if the visual presentation of the progress 
bar was still necessary. Reduction of visual cues could keep visual attention on the road and lead to safer driving.

\section{APPARATUS}

The experiment was conducted in a high-fidelity simulator using STI Sim ${ }^{2}$ software. The driving scenario was presented on a large screen and screens were integrated into as centre console and instrument cluster. The interaction and the simulator were controlled by two different desktop computers. Thermal feedback was provided by two Peltier pumps, $2 \times 2 \mathrm{~cm}$ in size, mounted on heat-sinks. Haptuator Mark $\mathrm{II}^{3}$ vibration actuators were taped onto each heatsink and attached to the back of the accelerator pedal. Heat-sinks were insulated by cork, so touching fingers would only feel the Peltier temperature. Devices were attached to the gear paddles with Velcro tape (see Figure 1).

\section{PARTICIPANTS AND PROCEDURE}

14 participants ( 3 female, 11 male), between 24 and 58 years (mean=38.21, STDEV=12.98) and all Jaguar Land Rover (JLR) employees, took part in the $1 \mathrm{~h}$ study. All held valid driving licenses for 3 to 41 years (mean=18.43, STDEV=12.33) and rated pre-experience with semi-autonomous features (median=4), driving simulator $($ median $=2)$, thermal feedback (median=1) and vibrotactile feedback (median=3.5) on 5-point Likert scale (1 no experience). The experiment took place at JLR labs, where participants read the information sheet and gave written consent, before driving. Ethics Committees of both JLR and the University of Glasgow approved the study.

\section{RESULTS}

\subsection{Gaze Behaviour and Time Observations}

Gaze behaviour and time observations evaluated by repeated measures ANOVA showed no significant differences (Overall eyes-offroad-time: haptic: $\mathrm{F}(1,26)=0.03, \mathrm{p}=0.86$; visual: $\mathrm{F}(1,26)=0.02$, $\mathrm{p}=0.90$ / Time-off-wheel: haptic: $\mathrm{F}(1,26)=0.02, \mathrm{p}=0.89$; visual: $\mathrm{F}(1,26)=1.41$, $\mathrm{p}=0.25$ / Time-off-pedal: haptic: $\mathrm{F}(1,26)=0.00, \mathrm{p}=0.96$; visual: $\mathrm{F}(1,26)=0.64$, $\mathrm{p}=0.43)$. Glances over $2 \mathrm{~s}$ were counted during handover and came to 3 glances overall (2 NoHapVis, 1 HapNoVis) by different participants. Those occurred when participants encountered interruptions of the transfer process, for example, by letting go of the paddles too early.

\subsection{Driving Behaviour}

Driving behaviour between conditions was evaluated with repeated measures ANOVA and showed no significant differences lane deviation or number of micromotions, for either condition. Driving within the conditions was evaluated with t-tests, if normally distributed, or Wilcoxon tests otherwise. Transfer type HapVis was the only condition with significant differences for lane deviation between baseline and handover: less lane deviation occurred when giving control to the car than during the baseline $(\mathrm{t}(13)=2.58, \mathrm{p}=0.023)$. Statistics of non-significant results are being omitted for brevity's sake.

\footnotetext{
${ }^{2}$ https://stisimdrive.com/

${ }^{3}$ http://tactilelabs.com/products/haptics/haptuator-mark-ii-v2/
}

\subsection{Subjective Rating}

Repeated measures ANOVA testing perceived workload showed no significant difference in overall workload for haptic $(F(1,26)=0.03$, $\mathrm{p}=0.87)$ or visual $(\mathrm{F}(1,26)=1.33, \mathrm{p}=0.26)$. Evaluation of additional ratings of pleasantness (haptic: $F(1,26)=0.02, p=0.90$; visual: $F(1,26)=1.05$, $\mathrm{p}=0.31$ ), comfort (haptic: $\mathrm{F}(1,26)=0.51, \mathrm{p}=0.48$ visual: $\mathrm{F}(1,26)=0.36$, $\mathrm{p}=0.56$ ), complexity (haptic: $\mathrm{F}(1,26)=0.07, \mathrm{p}=0.79$; visual: $\mathrm{F}(1,26)=0.00$, $\mathrm{p}=1.00$ ) and disruptiveness was only significant for disruptiveness for the factor visual $(\mathrm{F}(1,26)=10.50, \mathrm{p}=0.003)$, but not haptic $(\mathrm{F}(1,26)=0.24, \mathrm{p}=0.63)$. The lack of visual feedback increased the feeling of disruption. The ranking at the end of the experiment favoured HapVis over NoHapVis and lastly HapNoVis.

\section{DISCUSSION}

Time needed to disengage with the steering wheel and the acceleration pedal as well as overall eyes-off-the-road time was not significantly influenced by the feedback type, so addition of haptic feedback to the process did not improve this. However, the participants reported high familiarity with semi-autonomous features, which could have influenced this measure. They might already have been familiar with the transfer process, which would lead to less need for feedback. In future work, the modalities should be tested with participants unfamiliar with autonomous features. During activation, only transfer type HapVis showed significant reduction in lane deviation, but not number of micromotions. This shows that participants were keeping the vehicle within the middle of the road more steadily, while both visual and haptic feedback were presented during handover. This could indicate that participants felt reassured by the presentation of the additional haptic feedback and concentrate more on driving.

The subjective rating of the feedback showed no differences in reported workload, but there was a significant difference in reported disruptiveness: participants rated feedback without visual progress bar as significantly more disruptive than feedback with visual progress bar. Thermal feedback as sole presentation of the progress seems to not prepare drivers enough for the upcoming vibration, which was mirrored in the ranking: HapVis was rated highest, HapNoVis lowest.

\section{CONCLUSION}

We tested feedback combinations with haptic (thermal and vibrotactile) and visual feedback for control transfer between car and driver. While the influence on takeover will have to be explored further, the results for handover so far favour the use of haptic feedback with visual icons. The additional haptic feedback positively affected the driving performance during handover. Subjective ratings also showed a preference for visual and haptic feedback.

These promising results provide a basis for more insightful research into the effect of feedback modality on driver's preference and driving safety during control transfer in semi-autonomous cars.

\section{ACKNOWLEDGMENTS}

This research was supported by an EPSRC iCASE Award and Jaguar Land Rover (EP/P510506/1). 


\section{REFERENCES}

[1] Martin Albert, Alexander Lange, Annika Schmidt, Martin Wimmer, and Klaus Bengler. 2015. Automated Driving - Assessment of Interaction Concepts Under Real Driving Conditions. Procedia Manufacturing 3, Ahfe (2015), 2832-2839. https://doi.org/10.1016/j.promfg.2015.07.767

[2] Patrizia Di Campli San Vito, Stephen Brewster, Frank Pollick, Stuart White, Lee Skrypchuk, and Alexandros Mouzakitis. 2018. Investigation of Thermal Stimuli for Lane Changes. Proceedings of the 10th International Conference on Automotive User Interfaces and Interactive Vehicular Applications (2018), 43-52. https://doi.org/10.1145/3239060.3239062

[3] Patrizia Di Campli San Vito, Gözel Shakeri, Stephen Brewster, Frank Pollick, Edward Brown, Lee Skrypchuk, and Alexandros Mouzakitis. 2019. Haptic Navigation Cues on the Steering Wheel. CHI19 (2019), 1-11. https://doi.org/10.1145/ 3290605.3300440

[4] Christian Gold, Moritz Körber, Christoph Hohenberger, David Lechner, and Klaus Bengler. 2015. Trust in Automation - Before and After the Experience of Takeover Scenarios in a Highly Automated Vehicle. Procedia Manufacturing 3, Ahfe (2015), 3025-3032. https://doi.org/10.1016/j.promfg.2015.07.847

[5] S. G. Klauer, T. a. Dingus, V. L. Neale, J. D. Sudweeks, and D. J. Ramsey. 2006 The Impact of Driver Inattention On Near Crash/Crash Risk: An Analysis Using the 100-Car Naturalistic Driving Study Data. Analysis April (2006), 226. https: //doi.org/DOTHS810594

[6] Sebastiaan M. Petermeijer, Fabian Doubek, and Joost de Winter. 2017. Driver response times to auditory, visual, and tactile take-over requests: A simulator study with 101 participants. 2017 IEEE International Conference on Systems, Man and Cybernetics, SMC 2017 2017-Janua (2017), 1505-1510. https://doi.org/10. 1109/SMC.2017.8122827

[7] Sebastiaan M. Petermeijer, Paul Hornberger, and Ioannis Ganotis. 2018. The Design of a Vibrotactile Seat for Conveying Take-Over Requests in Automated
Driving. Advances in Human Aspects of Transportation 786 (2018). https://doi. org/10.1007/978-3-319-93885-1

[8] Ioannis Politis, Stephen Brewster, and Frank Pollick. 2017. Using multimodal displays to signify critical handovers of control to distracted autonomous car drivers. International fournal of Mobile Human Computer Interaction 9, 3 (2017), 1-16. https://doi.org/10.4018/ijmhci.2017070101

[9] Shadan Sadeghian Borojeni, Lewis Chuang, Wilko Heuten, and Susanne Boll. 2016. Assisting Drivers with Ambient Take-Over Requests in Highly Automated Driving. Proceedings of the 8th International Conference on Automotive User Interfaces and Interactive Vehicular Applications - Automotive'UI 16 (2016), 237244. https://doi.org/10.1145/3003715.3005409

[10] Katri Salminen and Ahmed Farooq. 2019. Unimodal and Multimodal Signals to Support Control Transitions in Semiautonomous Vehicles. In Proceedings of the 11th International Conference on Automotive User Interfaces and Interactive Vehicular Applications - Automotive UI'19. 308-318. https://doi.org/10.1145/ 3342197.3344522

[11] Gözel Shakeri, John H Williamson, and Stephen Brewster. 2017. Novel Multimodal Feedback Techniques for In-Car Mid-Air Gesture Interaction. Proceedings of the 9th International Conference on Automotive User Interfaces and Interactive Vehicular Applications - AutomotiveUI '17 (2017), 84-93. https://doi.org/10.1145/3122986. 3123011

[12] Sonja Stockert, Natalie Tara Richardson, and Markus Lienkamp. 2015. Driving in an Increasingly Automated World - Approaches to Improve the Driverautomation Interaction. Procedia Manufacturing 3 (2015), 2889-2896. https: //doi.org/10.1016/j.promfg.2015.07.797

[13] Kathrin Zeeb, Axel Buchner, and Michael Schrauf. 2015. What determines the takeover time? An integrated model approach of driver take-over after automated driving. Accident Analysis and Prevention 78 (2015), 212-221. https://doi.org/10. 1016/j.aap.2015.02.023 